Original Research Paper

\title{
Stress Analysis of an Artificial Human Elbow Joint: Application of Finite Element Analysis
}

\author{
Stephen K. Armah \\ Department of Mechanical Engineering, North Carolina A\&T State University, \\ 1601 E. Market Street Greensboro, NC 27411, USA
}

Article history
Received: 14-12-2017
Revised: 20-12-2017
Accepted: 03-01-2018

Email: skarmah@aggies.ncat.edu

\begin{abstract}
The elbow joint is frequently affected by two kinds of arthritis (degenerative arthritis and rheumatoid arthritis) following some kind of injuries. There have been efforts since the eighteenth century by various individuals and organizations in modeling permanent implants for the elbow joint, but some of the prostheses have been disappointing because of lack of understanding of the biomechanics of the elbow joint. This paper presents investigation of the stress analysis of the fixation of artificial elbow joint into the humerus bone of the human arm. The Finite Element Analysis (FEA) technique is used to study the stress distribution. The Amira software is used to develop the humerus bone model from a Computed Tomography (CT) data set. Then, the MSC Marc Mentat software is used to create implant and cement-mantle models from geometrical entities. The Magics software is used alongside to achieve appropriate models for the analysis. The maximum principal and von Mises stresses are obtained for varying lengths of the implant at a fixed diameter and with the elbow at an angle of fixation.
\end{abstract}

Keywords: Finite Element Analysis, Prosthesis, Implant, Artificial Elbow Joint, Fixation, Arthritis

\section{Introduction}

Many artificial joints are primarily and routinely used for the treatment of Rheumatoid Arthritis (RA) and rheumatoid osteoarthritis, especially hips and knees. Both diseases involve severe pain and prevent the sufferer from being able to move the joints freely, creating a major obstacle to daily life. The results of these diseases are mainly to destroy the articular cartilage lining on the articulating bone surfaces. This increases the friction and pain level due to bone rubbing on bone (Daripa et al., 2004). These diseases are particularly common in the elderly and can make it extremely difficult to perform basic activities such as walking, feeding, dressing, or even standing, preventing a normal life in society (Daripa et al., 2004).

In the US, doctor-diagnosed arthritis is a common and widespread chronic condition (Barbour, 2017). As the population ages, arthritis is expected to affect an estimated 1 in 4 (67 million) adults in the US by 2030 (Hootman and Helmick, 2006). From 2013-2015, an estimated 54.4 million US adults (22.7\%) annually had ever been told by a doctor that they had some form of arthritis; RA, gout, lupus, or fibromyalgia (Barbour, 2017).
For a long time, different surgical operations have been proposed to restore joint mobility and stability; involving resection of the articulating bone end parts. However, one-third of such operations are proved to be unsatisfactory (Daripa et al., 2004). Elbows are not replaced very often, largely because their fixation has not always been reliable and very little work has been done on elbows in the past, compared to the other joints. About 500,000 knee replacements and more than 175,000 hip replacements are performed annually and those numbers are on the rise. In fact, hip replacements are expected to increase $174 \%$ in the next 20 years and knee replacements will rise even more (Shaw, 2017). Shoulder replacements are less frequent and elbow replacements are very seldom performed (Tidy, 2017). The elbow joint is estimated to be involved in 20 to $65 \%$ of the patients with RA, although only $5 \%$ of the patients develop isolated elbow involvement (Studer and Athwal, 2011; Sanchez-Sotelo, 2016).

Today, more than 200 years after the first production of metallic magnesium by Sir Humphry Davy in 1808, biodegradable magnesium-based metal implants are currently breaking the paradigm in biomaterial science to develop highly corrosion resistant metals (Witte, 2015). 
This ground-breaking approach to temporary metallic implants is one of the latest developments in biomaterials science that is being rediscovered (Witte, 2015). It is a challenging topic and several secrets remain that might revolutionize various biomedical implants currently in clinical use (Witte, 2015). These non-permanent, biodegradable implants have been in clinical use for nearly three decades, competing directly with permanent implants (Amini et al., 2011). Biodegradable orthopedic implants, have their associated short- and long- term inflammatory effects, (Amini et al., 2011). Although traditional permanent implants have their drawbacks, they have been proved clinically efficacious.

There have been efforts since the eighteenth century by various individuals and organisations in modeling permanent implants for the elbow joint, but some of the prostheses have been disappointing because of lack of understanding of the biomechanics of the elbow joint. The elbow joint is a complicated anatomy of the human body and for adequate design of an implant for the joint, there is the need of in-depth understanding of the forces and stresses that comes into action as a result of the functioning of the arm.

The permanent implants currently being used in the surgical profession do not last long because of simplification of the biomechanics of the joint in the design. One of the problems of the latest designs is the issue of suitable length of the implant stem into the humerus, ulna and radius bones, since it can give a lot of complication to the patients. A well-designed artificial implant should cause no harm to the body, last long and should be able to fix to the existing bones. Such implants will go a long way to give the desired relief of the pain and positive psychological benefits to the patients. This calls for more understanding of the forces and stresses at the joint for better and adequate designing of artificial elbow joints and hence the work in this study.

During the last six decades, there has been a tremendous increase of interest in the development of a hinged total elbow prosthesis; these include those designed by Coonrad, Dee, Gschwend and Scheier and Bähler (the G.S.B. prosthesis), McKee, Pritchard, Engelbrech (the St. George prosthesis), Schlein, Shiers, Scales (the Stanmore prosthesis) and Swanson. Most of these implants are made of metal only, but some, such as the Swanson and Coonrad implants, have an interface of polyethylene to prevent metallic wear and subsequent synovitis. Many have not yet had an adequate clinical trial (Completo et al., 2011).

Total Elbow Arthroplasty (TEA) is a surgical procedure performed to restore the functions affected by RA. There are two common types of permanent elbow implants used in the current medical field for TEA (Khoo et al., 2006). They are the linked, semiconstrained elbow implant and the unlinked, unconstrained elbow implants (Khoo et al., 2006). One of such implants is the Nakashima's elbow joint prosthesis shown in Fig. 1. It is a product of Nakashima's medical equipment, comprised of a humeral component made of titanium alloy and an ulna component made of ultrahigh molecular weight polyethylene. They are reconstructed by sliding the articular surfaces of these two components (EJP, 2017). To allow free movement, the artificial joint is carefully designed to exactly replicate the natural articular surfaces of the elbow (EJP, 2017). Depending on the product, they can offer a radial component that enables the elbow to bend and stretch naturally (EJP, 2017).

Another permanent implant, which is widely used in the orthopaedics industry and currently the world's number one selling implant, is the Coonrad/Morrey total elbow implant, Fig. 2. It comprised of a Ti6Al4V alloy humerus and ulna components and polyethylene bushings to prevent metal-to-metal contact. The surfaces of the humerus and ulna components are triangular and quadrangular to match the shapes of the humerus and the ulnar canals, respectively (EP, 2017). Portions of the surfaces of the stems are coated with plasma spray to enhance the cement fixation (EP, 2017). The humerus stems comes in the lengths of 4,6 or 8 inches and the ulnar stems in regular or longer lengths.

Bone has a high compressive strength of about 170 $\mathrm{MPa}$ and poor tensile strength of 104-121 MPa and a very low shear stress strength of 51.6 MPa. This means that bone resists pushing (compressional) stress well, resist pulling (tensional) stress less well, but only poorly resists shear stress (such as due to torsional loads). While bone is essentially brittle, bone does have a significant degree of elasticity, contributed chiefly by collagen. In normal bone, fractures occur when there is significant force applied, or repetitive trauma over a long time. Fractures can also occur when a bone is weakened, such as with osteoporosis, or when there is a structural problem, such as when the bone remodels excessively (such as Paget's disease) or is the site of the growth of cancer (Bone, 2017). It has been reported in the literature that permanent elbow implants can induce peak stress values between 100-200 MPa on the elbow joint bones for a joint load of $1.0 \mathrm{kN}$. Also, a joint load between 1.2-3.0 kN is expected to cause failure of the humerus bone initiated from the location of the peak maximum principal stress.

This paper presents investigation of the stress analysis of the fixation of permanent artificial elbow joints into the humerus bone of the human arm. The Finite Element Analysis (FEA) technique is used to study the stress distribution. The Amira software is used to develop the humerus bone model from a Computed Tomography (CT) data set. Then, the MSC Marc Mentat software is used to create implant and cement-mantle models. The Magics software is used alongside to achieve appropriate models for the analysis. The maximum principal and von Mises stresses are obtained for varying lengths of the implant at a fixed diameter and with the elbow at an angle of fixation. 


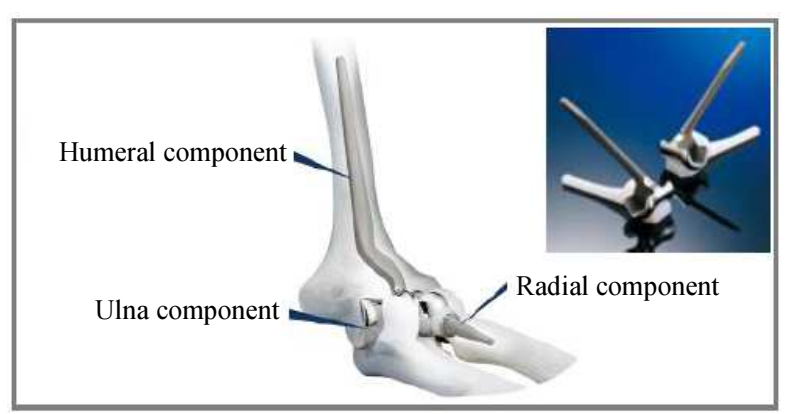

Fig. 1: Nakashima's elbow joint prosthesis (EJP, 2017)

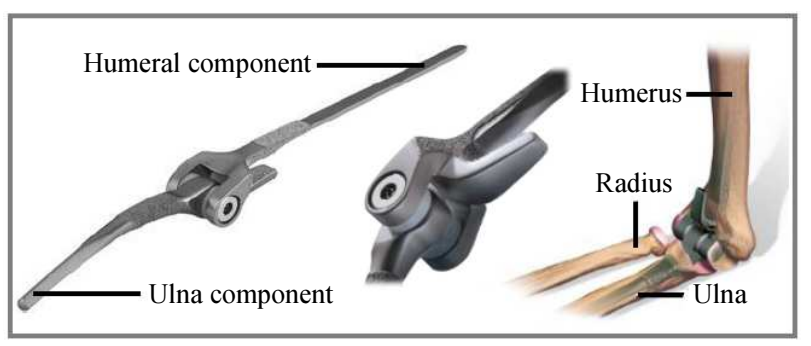

Fig. 2: The Coonrad-Morrey total elbow implant (EP, 2017)

This paper is organized as follows. Section II presents the biomechanics of the elbow-forearm complex. In section III, FEA procedure is discussed. The methodology applied in the development of the FEA humerus bone, implant and cement mantle models are presented in section IV. In section $\mathrm{V}$, results are summarized. Concluding remarks and future work are presented in section VI.

\section{Biomechanics of the Elbow Joint}

\section{The Anatomy of the Elbow Joint}

The elbow or elbow joint is an anatomic hinge-joint (ginglymus) and a trochoid-joint or what is termed as trochoginglymoid joint, which connects the radius-ulna of the forearm and the humerus of the arm for the movement of the mechanical chain that begins at the shoulder and ends at the fingertips, Fig. 3. It comprises of three joints, whose articulate surfaces are connected together by a capsule, namely: (1) Humerus and ulnar or the humeroulnar (hinge-joint), (2) Humerus and radius or the humeroradial (hinge-joint) and (3) Proximal radioulnar (trochoid-joint).

The shoulder, as the first link, functions to permit the hand to be positioned anywhere within an imaginary sphere that represents the full excursion of shoulder motion. Elbow motion allows the height and length of the upper extremity to be adjusted, whereas forearm rotation allows the hand to be placed in the most effective position for function.

\section{Kinematics of the Elbow Joint}

The understanding of the kinematics of the elbow joint is a requirement for elbow joint replacement, the upper limb prostheses, or arthroses, so that realistic set and adequate design for the artificial devices can be developed. There are different ranges of motion which are normally recognized at the elbow, but the two which allows the joint to have two degrees of freedom in motion are: (1) Flexion-extension and (2) Pronation-supination, Fig. 4. In fact, the motion at the elbow is a complex threedimension, with a third motion describe as the passive carrying angle (Wadsworth 1982; Khoo et al., 2006; Amis and Miller, 1981). This is the motion about the $x$ axis at any position in the $y z$-plane.

\section{The Kinetics of the Elbow Joint}

The flexion-extension and pronation-supination motions at the elbow joint is because of the actions of the muscles surrounding the elbow. Each specific motion generally results from the action of more than one muscle; furthermore, some muscles participate in producing more than one specific motion. Analyses have shown that one or more of about fourteen muscles produce force at the elbow joint during articulation of the joint (Zuckerman and Matsen, 1989). More often in the design of joint replacement, only about two or three of these muscles are considered to be acting, leading to underestimation of the forces at the elbow (Zuckerman and Matsen, 1989). Most of the muscles involved in elbow function and stability originate on the humerus and insert on either the radius or ulna. Humeroradial muscles include the biceps, brachioradialis, and pronator teres.

A lot of work has been done, using different techniques, in measuring muscles forces. The use of electromyographic data in (Basmajian and Latif, 1957), to determine that the brachialis, which arises from the anterior aspect of the humerus and inserts on the anterior aspect of the proximal ulna, was the primary flexor of the elbow. The hypothesis that muscle forces may be related to the cross-sectional area of fibres within the muscles during maximal activities was used to analysed lower limb forces (Alexander and Vernon, 1975). Based on the above results, the equal stress hypothesis, for groups of co-operating muscles, was used as the initial basis to study the maximal actions at the elbow joint and the upper limb musculature to published data on relative muscle strengths, (Amis et al., 1979; 1980a).

\section{Static and Dynamic Effects on Elbow Joint Forces}

The reaction force on the ulna by the humerus and the force produced through the tendons of the muscle in certain static and dynamic situations can be estimated at various flexed angles of the elbow joint, if some assumptions are made. As an example on a static situation, consider the free-body diagrams shown in Fig. 5. 


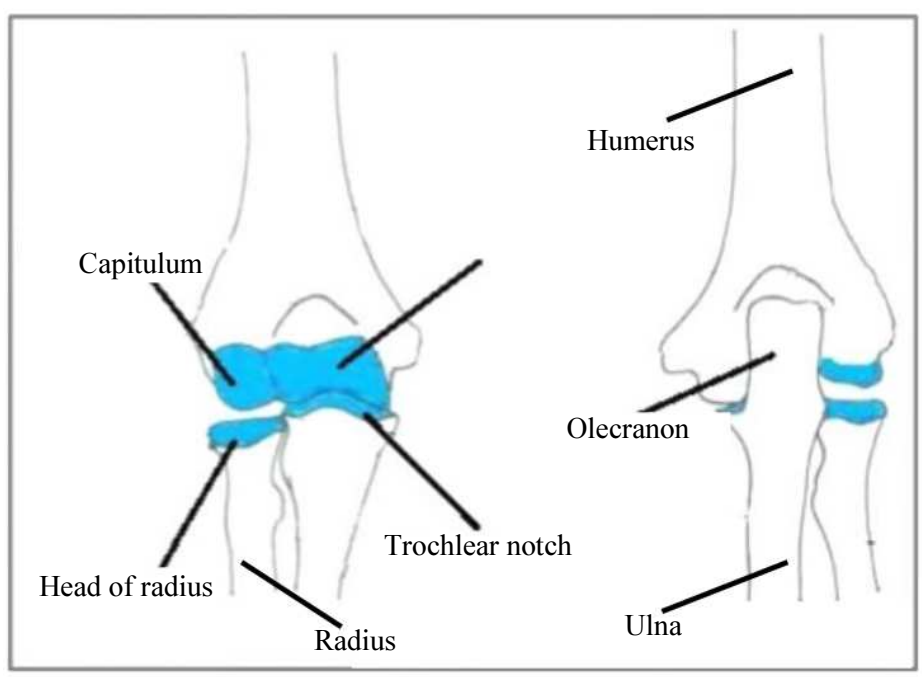

Fig. 3: Anterior and posterior view of the elbow joint (EJ, 2017)

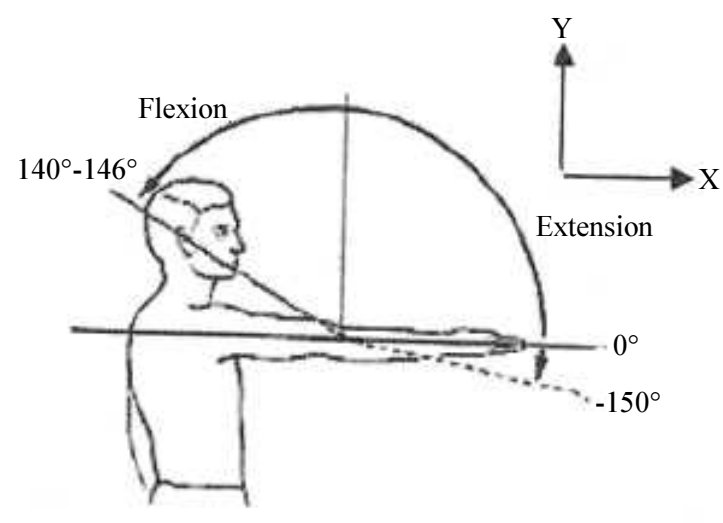

(a)

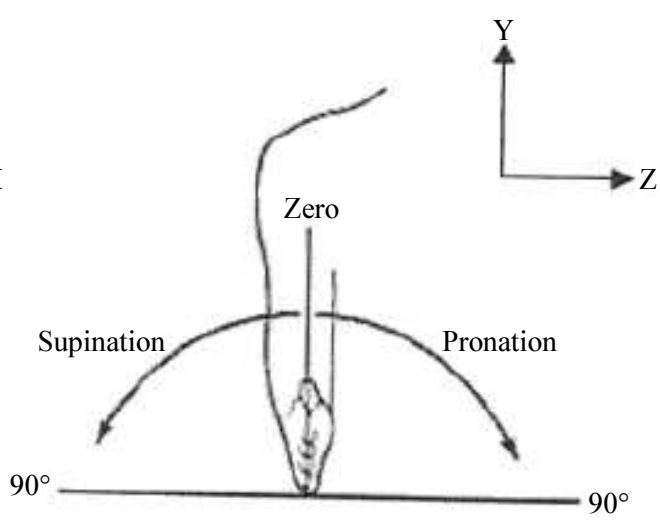

(b)

Fig. 4: (a) Flexion-Extension (b) Pronation-Supination Motion at the elbow Joint (Sanchez-Sotelo, 2016)
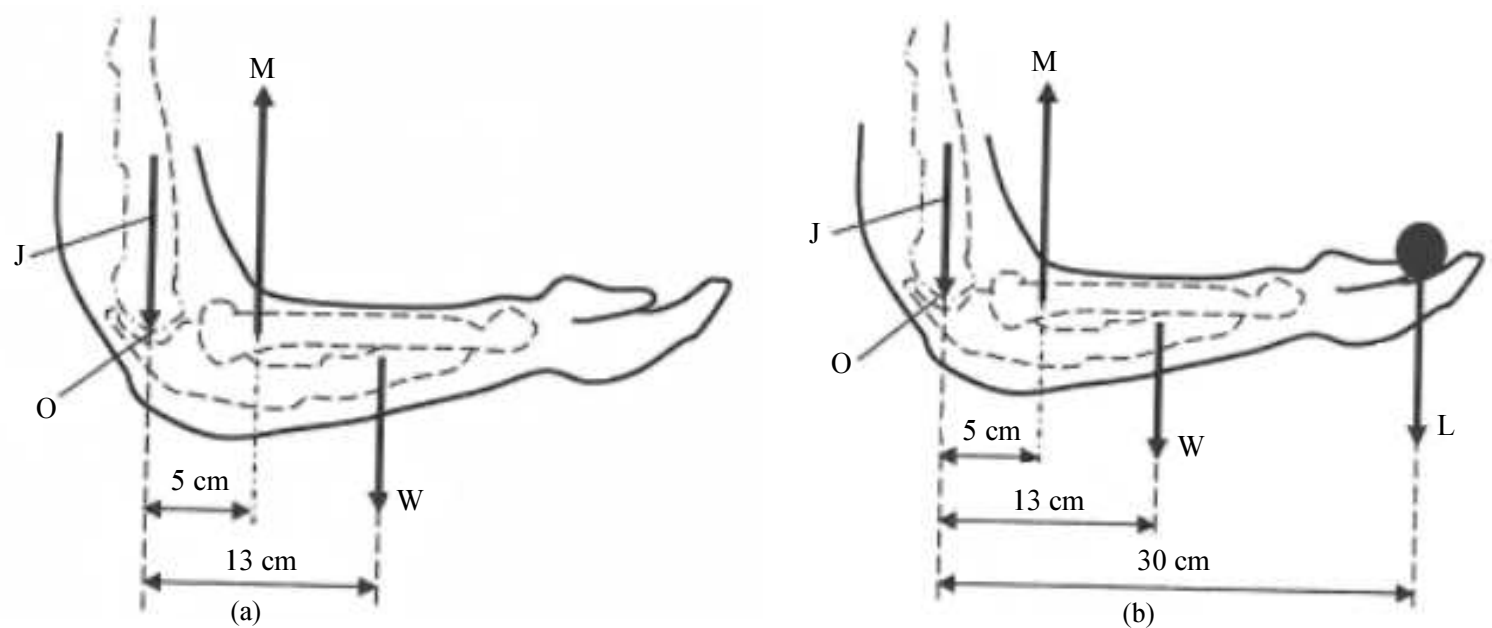

Fig. 5: (a) No weight in hand (b) Weight in hand. Reaction force on the ulna and the force muscle with the elbow flexed at $90^{\circ}$ (Amis and Miller, 1981) 
If it is assume that (1) elbow flexed at $90^{\circ}$, (2) the predominant flexors are brachialis and biceps, (3) the force through the tendons of the muscle act at $90^{\circ}$ to the forearm axis and (4) the dimensions and weight $(W=20$ $\mathrm{N}$ ) of the forearm are as indicated. Then, the reaction force on the ulna by the humerus and the force through the muscles, $J$ and $M$, respectively, could be estimated from the diagram. If the external load $(L)$ in the hand is taken as $1 \mathrm{~kg}$, Fig. $5 \mathrm{~b}$, then $M$ and $J$ can be computed as follows:

Taking moments about $O$ :

$(13 \mathrm{~cm} \times W)+(30 \mathrm{~cm} \times L)=(5 \mathrm{~cm} \times M)$

Summing forces in the vertical direction:

$M=J+W+L$

giving $M=111 \mathrm{~N}$ and $J=81 \mathrm{~N}$. Note that, if $L=0$, Fig. $5 \mathrm{a}$, then $M=52 \mathrm{~N}$ and $J=32 \mathrm{~N}$.

Various work have been done in the literature about the dynamic effects on the elbow joint forces, (Hollerbach and Flash, 1982; Lawrence and Gerald, 1963). The purpose of some of the earlier research work was as a result of anticipation of higher forces due to the dynamic effects than those developed in the static situation (Amis et al., 1980b). The joint forces were analyzed at high-speed forearm movements, usually for the purpose of considering muscle mechanics. In (Amis et al., 1980b), the forces were analysed kinematically due to high-speed flexion and extension of the forearm, through the full range movement. It was derived that, the moment, $M_{o}$, about elbow flexion axis is given as:

$$
M_{o}=2.4095 \cos \theta+0.00599 \ddot{\theta}
$$

where, $\theta$ is the angle flexion.

This equation has been used to estimate the joint forces caused by the major muscle actions for various angle of flexion. It was observed that movements lasting $0.25 \mathrm{~s}$, angular velocities of $18 \mathrm{rad} / \mathrm{s}$ and angular accelerations of $570 \mathrm{rad} / \mathrm{s}^{2}$ were seen. An initial acceleration peak was normally followed by a prolonged constant-torque phase (Amis et al., 1980b). The motion was arrested abruptly: The fastest movements sometimes caused decelerations of $1100 \mathrm{rad} / \mathrm{s}^{2}$ (Amis et al., 1980b). It was shown that the muscles alone might produce such decelerations. Analysis of elbow joint forces during these actions suggested that the articulations were not subjected to forces beyond those seen during maximal isometric efforts (3.2 kN maximum) (Amis et al., 1980b).

\section{The Fixation of the Artificial Elbow Joints}

Elbow implant arthroplasty is a difficult technical and surgical procedure, which closely follow a prescribed plan. An accurate knowledge of the regional anatomy and experience in performing prosthetic replacement are required. Currently, there are many organizations such as Coonard-Morrey Total Elbow, Life Extension Foundation, Nakashima Propeller Company producing elbow implants for the orthopaedics industry, but the procedure employed in artificial elbow joints fixation generally involves indications or contraindications, preoperative considerations, surgical technique and postoperative management. The surgical technique involves the following steps summarized here and shown in Fig. 6 (EP, 2017; Schneeberger et al., 2007):

- Incision of the elbow

- Humeral resection

- Preparation of the ulna

- Trial reduction

- Cement technique

- Humeral bone graft

- Assembly and impaction

- Closure

\section{Finite Element Analysis}

FEA is a numerical technique for solving problems of engineering and mathematical physics (FEM, 2017). The problems are the field equations of a body. It is also referred to as Finite Element Methods (FEM). Typical problem areas of interest include structural analysis, heat transfer, fluid flow, mass transport and electromagnetic potential (FEM, 2017). The analytical solution of these problems generally require the solution to boundary value problems for partial differential equations (FEM, 2017). The finite element method formulation of the problem results in a system of algebraic equations (FEM, 2017).

In the context of structural analysis, the method is used to calculate the displacements and stresses at any (or all) locations of the structure when loading is applied. In the latter half of last century rapid progress in all engineering fields has been attributed to the implementation of numerical solution schemes. The first to appear was the FEA, which although initially confined to the major industries, i.e., aircraft and automotive, soon became adopted by the rest of the engineering community as the cost of computer hardware and software decreased. General purpose FE software began to appear in the 1970 s and by the late 1980 s commercial FE packages were available on microcomputers with color graphic pre- and post-processors (Kim, 2014).

Finite element formulation are based around discretizing the geometry domain into a number of small regions or (finite) elements, connected at nodes, which are like mathematical pins holding elements together. This process of subdivision results in a set of algebraic equations. These equations are the equilibrium equations for the nodes. These system of linear equations is constructed in matrix form as:

$[K]\{u\}=\{F\}$ 


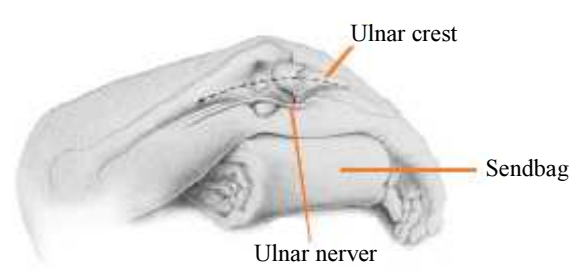

(a)

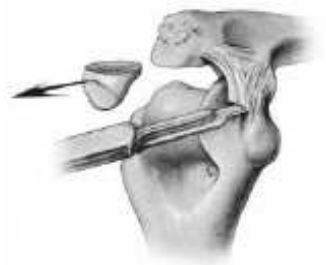

(b)

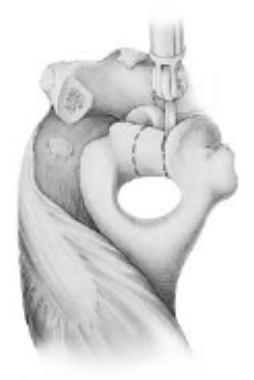

(c)

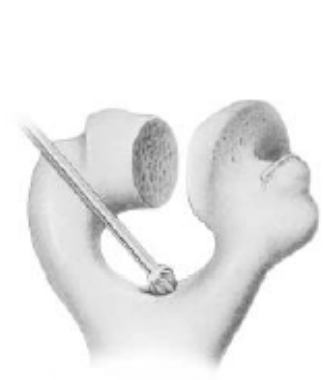

(d)

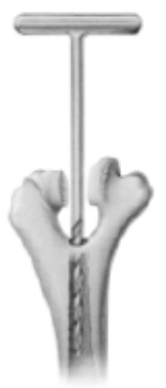

(e)

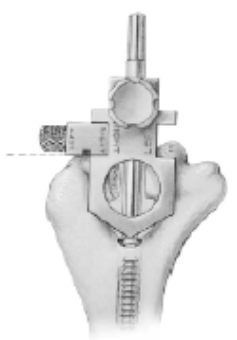

(f)

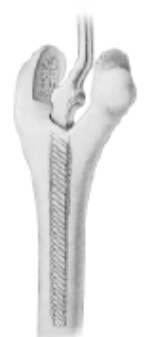

(g)

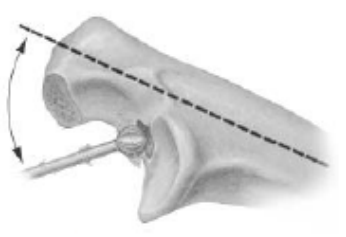

(h)

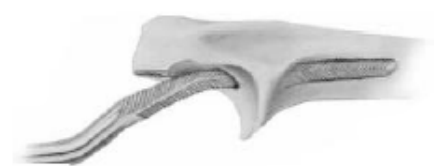

(i)

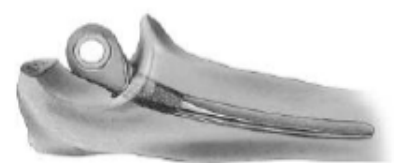

(j)

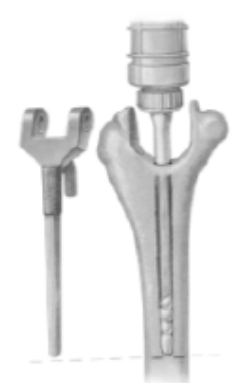

(k)

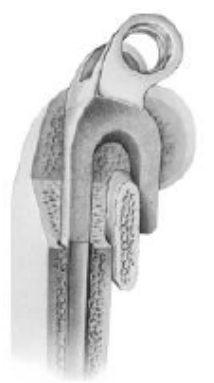

(1)

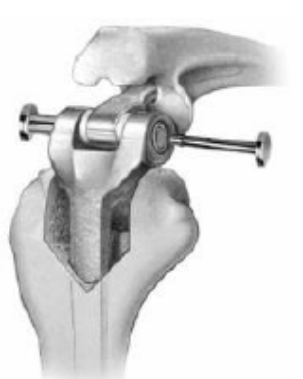

(m)

Fig. 6: Steps involve in artificial elbow joints fixation (EP, 2017; Schneeberger et al., 2007)

where, $[K]$ is the overall (global) stiffness matrix, $\{u\}$ is an unknown vector of field quantity such as displacement at nodes and $\{F\}$ is the vector of known applied loads (Kim, 2014). Then, material properties are assigned to each of element and appropriate loading and boundary conditions are defined. Finally, the combined governing equations for each element are solved using iterative methods. Over an element, a field quantity is interpolated from values of the field quantity at nodes (Kim, 2014). A calculated solution can be inaccurate if the solution has not converged. Therefore, a convergence test is conducted to obtain accurate solution. 
FEA was adopted in the field of orthopaedic biomechanics in 1972 to calculate stresses in a human body (Brekelmans et al., 1972). Improvements in computer technology have allowed for the solution of increasingly complicated FE models. However, the generation of FE models of anatomical structures from CT data, particularly for use within orthopaedic related biomechanical analyses, remains a difficult challenge. One of the major drawbacks with the use of the FEA in the field of orthopaedic biomechanics is the complexity associated with the development of realistic and manageable models of anatomical data, protocols for generating FE models, assignments of material properties, the application of suitable boundary condition to the structure, simulating loading upon the structure and incorporating prostheses within the anatomy all have to be considered carefully (Hopkins et al., 2007).

\section{Development of FEA Models}

This section presents the development of the FEA humerus bone, implant and cement-mantle models.

\section{Humerus Bone Model Generation}

The FE humerus bone model was generated using the segmentation feature of the Amira software. A data set of CT scan, a test bone, which consisted of set of 39 slides or sections of the humerus bone was acquired. The generation process involved the following steps:

- Loading the data set

- Threshold segmentation

- Refining segmentation results

- Extracting surfaces from segmentation results

- Simplifying the surface

- Generation of a tetrahedral grid

Figure 7 shows the final humerus model, $135 \mathrm{~mm}$ length, of the tetrahedral elements model generated.

\section{Generation of Implant and Cement-Mantle Models}

The MSC Marc Mentat software geometrical meshing technique was applied in generating the implant and cement-mantle models. Initially the 3-inch (76.2 $\mathrm{mm}$ ) implant model was made. A surface mesh was initially built in the first quadrant of the $x y$-plane by first constructing circular arc geometrical entities starting from radius $1 \mathrm{~mm}$ to $5 \mathrm{~mm}$ at intervals of $1 \mathrm{~mm}$ for the implant-stem and from $5 \mathrm{~mm}$ to $6.5 \mathrm{~mm}$ at intervals of $0.5 \mathrm{~mm}$ for the cement-mantle. Then, using symmetry, the quarter surface mesh was reflected first about the $y$ axis then about the $x$-axis to generate the full surface mesh, Fig. 8. The surface model template developed was the translated in the $z$-axis direction about it origin, for 10 repetitions of $62.5 \mathrm{~mm}$ length, to developed the 3D solid mesh shown in Fig. 9.

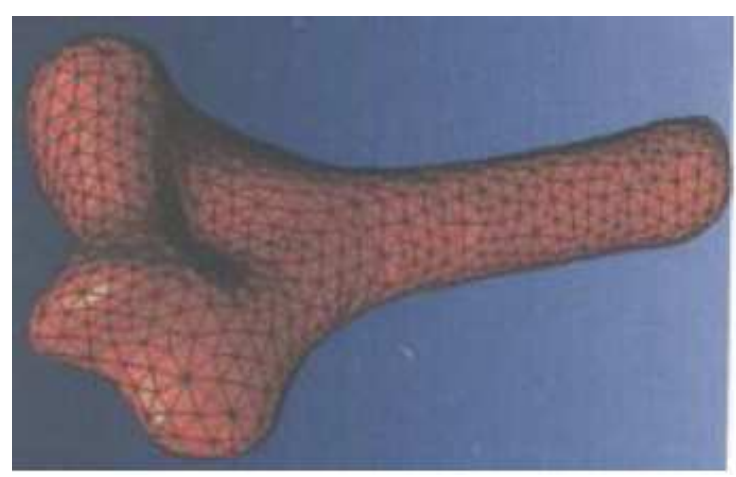

Fig. 7: Smoothed surface of the tetrahedral elements model generated

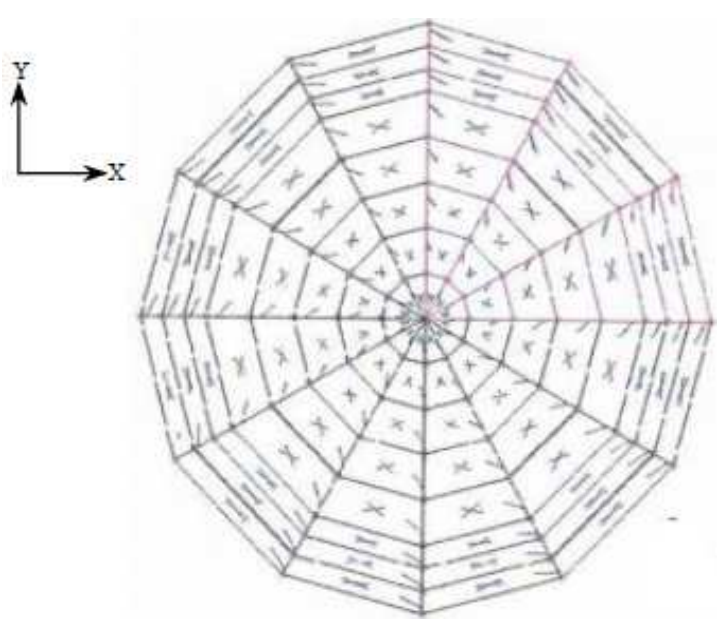

Fig. 8: Implant-stem and cement-mantle surface mesh

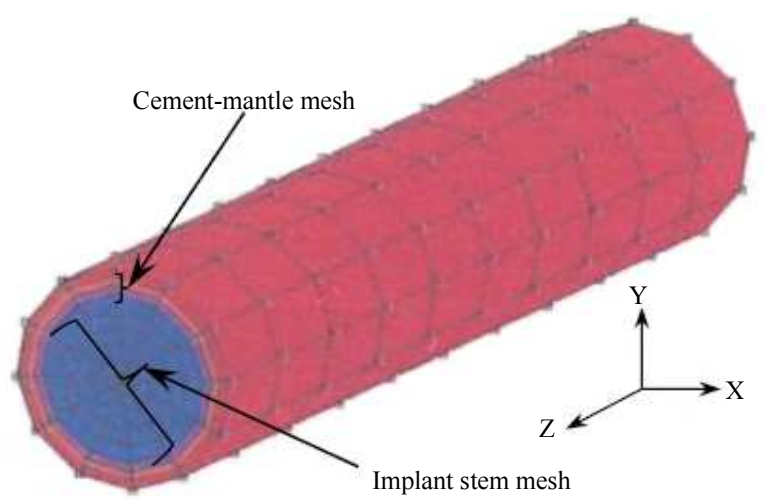

Fig. 9: Combined implant-stem and cement-mantle mesh

The curved portions of the implant-stem and cementmantle models were developed separately from the same surface mesh template. For example, the curved portion of the cement-mantle was made by first removing the implant set from the original surface model template and then rotated through $\left(0^{\circ}, 5^{\circ}, 0^{\circ}\right)$ for 4 repetitions about the point $(-29,0,0)$, Fig. 10 . The curved-implant stem model developed is shown in Fig. 11. Since there must 
be a cement-mantle at the bottom of the humeral cavity, a cement of $1.5 \mathrm{~mm}$ thickness was developed. This was done by translating the original surface model template in the $\mathrm{z}$-axis direction about it origin, for 1 repetition of $1.5 \mathrm{~mm}$ length, Fig 12. The portions of the implant-stem and cement-mantle developed were then merged together, Fig. 13.

Figure 14 shows the implant-head mesh model. The surface template for this model was generated in the same way as the one for the combined implant-stem and cementmantle surface mesh. In this case, it was developed in the $x z$-plane and the circular arc geometrical entities started from a radius of $1.5 \mathrm{~mm}$ to $15 \mathrm{~mm}$ at intervals of $1.5 \mathrm{~mm}$. The implant-stem and cement-mantle models are first merged and then merged with the implant-head to create a Hex mesh, unified model, Fig. 15.

To unify the implant-stem and the implant-head, a model consisting of the merged implant-stem and implanthead was created by removing the cement-mantle set from the model, Fig. 16. The implant-stem-implant-head model was then exported into the Magics software as STL (stereolithographic) file, by first converting the solid mesh into triangular surface mesh in the Mentat. The file was then loaded in Magics and the unified to make the model as one surface mesh. This procedure comes with a problem as the distribution of the triangular elements can be damaged, especially around the contour of the intersection, as very small triangular surfaces are formed. This normally gives problem when converting the surface mesh back to solid mesh in Mentat. The problem was solved by removing smaller elements and creating larger triangular elements around the intersection contour.

Further problem developed in the solid mesh generation from the surface mesh, as the model contains some free corners or edges and therefore was taken back and forth until it was free from this problem. The repaired surface model was finally loaded in Mentat and then a tetrahedral elements solid mesh was created to generate the 3-inch implant model, Fig. 17. The implant has a stem diameter of $10 \mathrm{~mm}$ with a head diameter of $30 \mathrm{~mm}$ and thickness of $16 \mathrm{~mm}$ and the cement-mantle model has a thickness of $1.5 \mathrm{~mm}$.

\section{Cutting of the Humerus Bone Model Canal}

This cut-out model was generated in the same way as the implant model, by exporting as STL file into Magics for the unification of the implant-stem and the implanthead, but in this case the cement-mantle was attached, Fig. 18. The humerus bone and the cut-out models were loaded and aligned to the desired position using the Amira software as shown in Fig. 19. A file of the aligned model was then loaded in Magics software. Using Boolean tools within software, the canal was cut-out from the humerus bone model. The surface mesh of the cut-out humerus bone model was then converted to tetrahedral solid mesh using similar procedure discussed in previous text, Fig. 20.

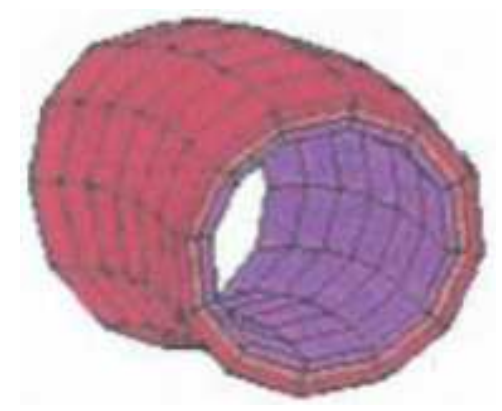

Fig. 10: The curved portion of the cement-mantle mesh

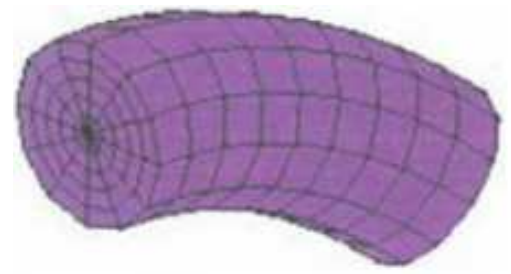

Fig. 11: The curved portion of the implant stem mesh

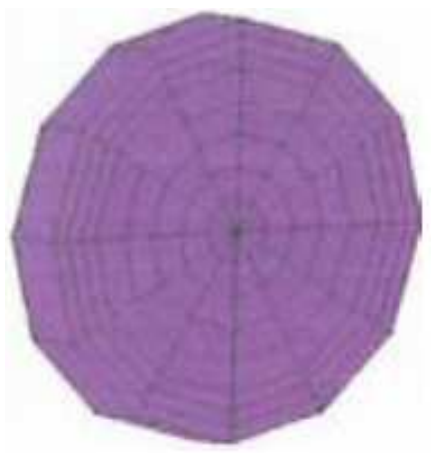

Fig. 12: The bottom cement-mantle mesh

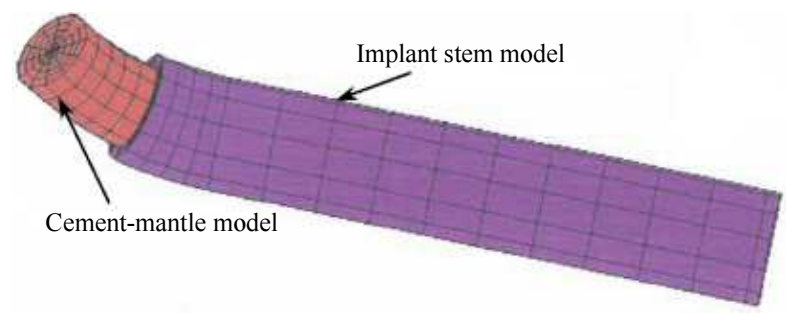

Fig. 13: Merged implant-stem and cement-mantle mesh

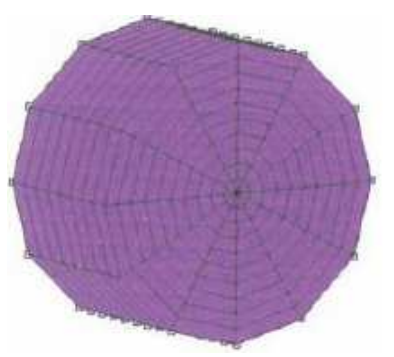

Fig. 14: The implant-head mesh 


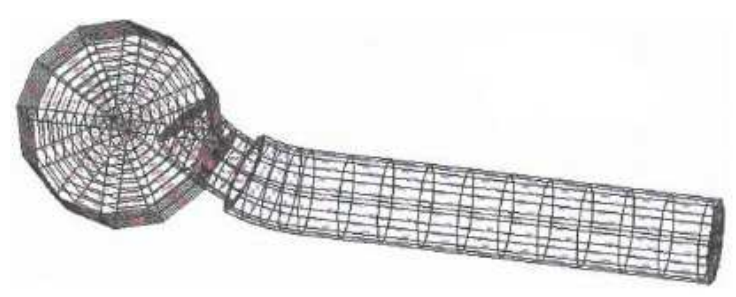

Fig. 15: Merged implant-head, implant-stem and cement-mantle (Hex mesh, not-unified)

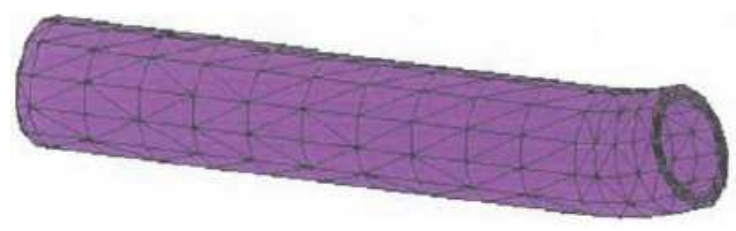

Fig. 16: The cement-mantle mesh model

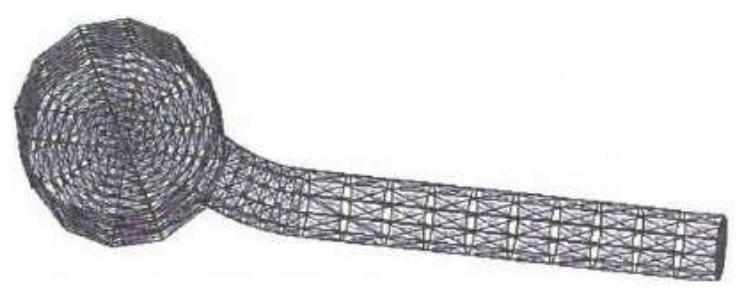

Fig. 17: Tetrahedral solid model of the 3-inch implant

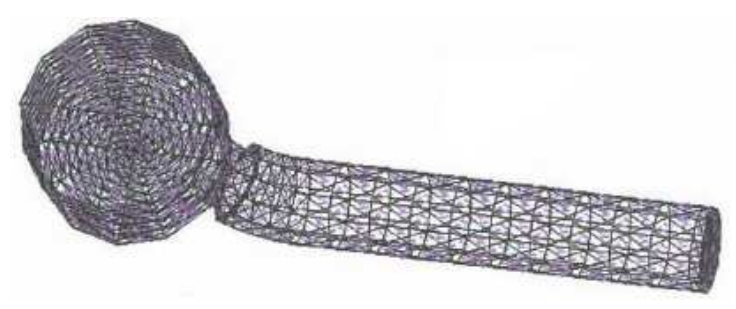

Fig. 18: The cut-out surface mesh
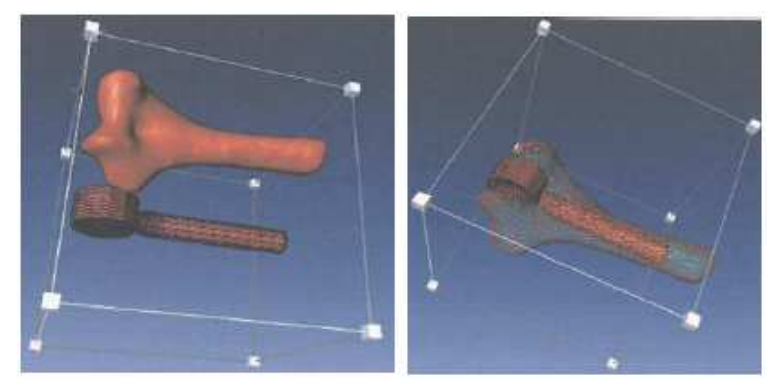

Fig. 19: The alignment humerus bone model and cut-out models

\section{Merging of the Three Models}

After all the three solid tetrahedral elements have been developed, they were loaded in Mentat and merged for the continuation of the FEA, Fig. 21.

\section{The 2-Inch and 4-Inch Models Developed}

The same procedure described above was used to develop the 2-inch $(50.8 \mathrm{~mm})$ and 4-inch $(101.6 \mathrm{~mm})$ implant models, cement-mantle models, their corresponding humeral cut models, Fig. 22 and 23. Table 1-3 give some data of the models developed.

\section{Boundary Conditions}

The following mechanical boundary condition was applied, with the same time load history applied to merged model (Fig. 24): (1) Fixed displacement was applied on the humerus bone model at the truncated section of the stem, to support the whole structure as a cantilever (2) Four point loads were applied on the merged model: (a) Lateral Condyle Load $\left(F_{L C}\right)$, (b) Medial Condyle load $\left(F_{M C}\right)$, (c) brachialis muscle load $\left(F_{B M}\right)$ and $(\mathrm{d})$ joint load $(J)$. The muscle force ratios were taken from a table in (Witte, 2015). An external load $(L)$ of $67 \mathrm{~N}$ was applied:

$$
\begin{aligned}
F_{L C}= & \text { Lateral epicondyle force }\left(F_{L E}\right)+ \\
& \text { Brachioradialis force }\left(F_{B}\right)
\end{aligned}
$$

where $F_{L E}=3.5 L$ and $F_{L E}=1.93 L$, thus $F_{L C}=363.81 \mathrm{~N}$ $\approx 0.365 \mathrm{kN}$. This was applied at a node on the lateral epicondyle at an angle of $60^{\circ}$, with respect to the axis of the humerus bone model:

$$
\begin{gathered}
F_{M C}=\text { Medical epicondyle force }\left(F_{M E}\right)+ \\
\text { Pronatorteres force }\left(F_{P T}\right)
\end{gathered}
$$

where $F_{M E}=1.25 L$ and $F_{P T}=3.17 L$, thus $F_{M C}=296.14$ $\mathrm{N} \approx 0.3 \mathrm{kN}$. This was applied at a node on the medial epicondyle at an angle of $60^{\circ}$, with respect to the axis of the humerus bone model. $F_{M B}=6.15 L$, thus $F_{B M}=$ $412.05 \mathrm{~N} \approx 0.415 \mathrm{kN}$. This was applied at a node on the anterior aspect of the humerus model at an angle of $43^{\circ}$, with respect to the axis of the humerus bone model. The joint load, with an approximate value of $1 \mathrm{kN}$, was applied at a node on the implant-head at an angle of $38^{\circ}$ with respect to the axis of the humerus bone model. The elbow flexed at $60^{\circ}$ to obtain the approximated external load of $0.067 \mathrm{kN}$ in the hand. The applied point loads are summarized in Table 4.

\section{Material Properties}

The Mentat program recognizes many material data, which include: Isotropic, Orthotropic, Anisotropic, Hypoelastic, Mooney, Ogden, Foam, Soil, Powder, Heat transfer, Joule heating, Acoustic, Bearing, Electrostatic, Magnetostatic, and Electromagnetic. All the three models were assigned isotropic elastic material properties as shown in Table 5. 

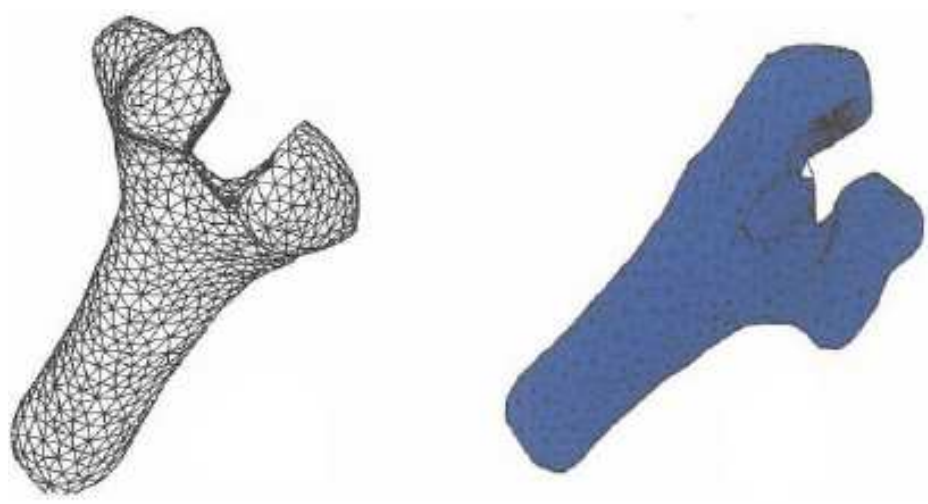

Fig. 20: The cut-out humerus bone solid mesh
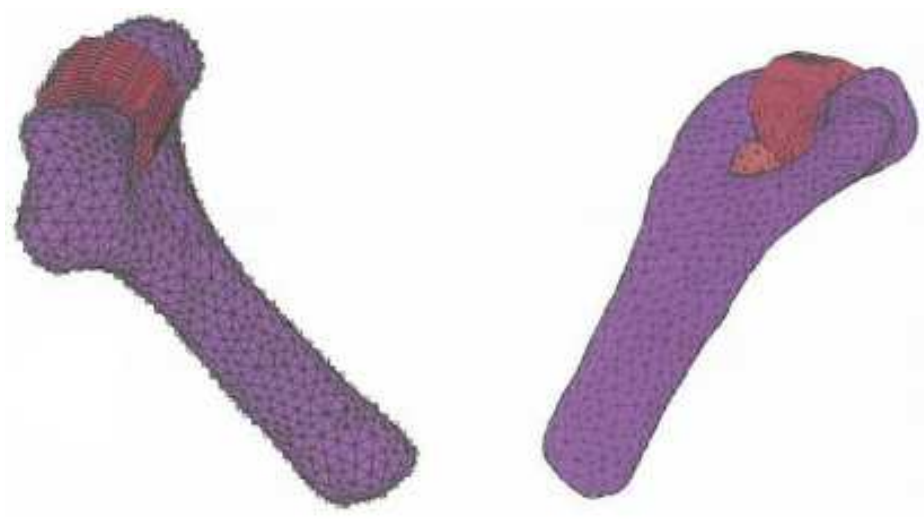

Fig. 21: Merged of the three models

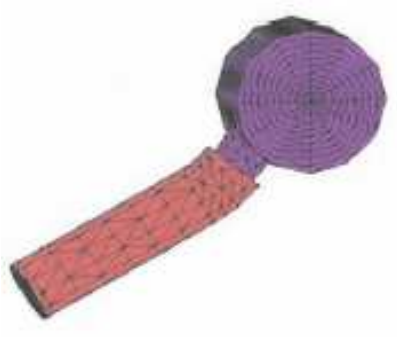

(a)

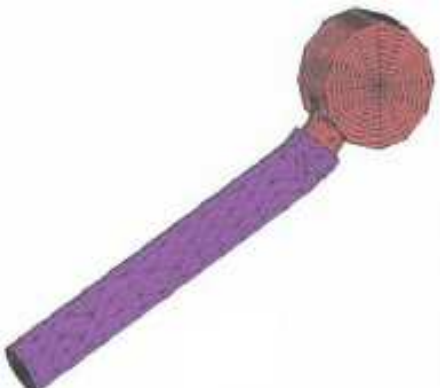

(b)

Fig. 22: (a) 2-inch merged implant and cement-mantle model (b) 4-inch merged implant and cement-mantle model

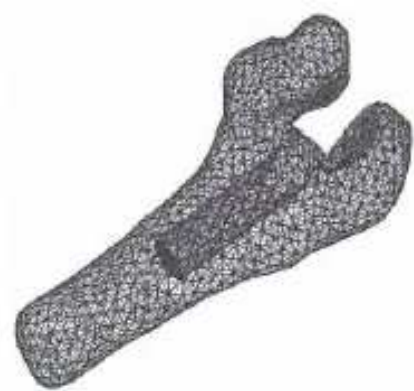

(a)

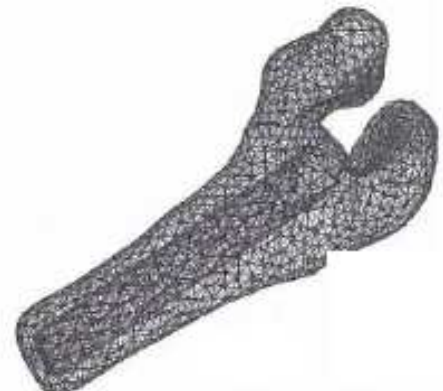

(b)

Fig. 23: (a) 2-inch cut-out humerus bone model (b) 4-inch cut-out humerus bone model 


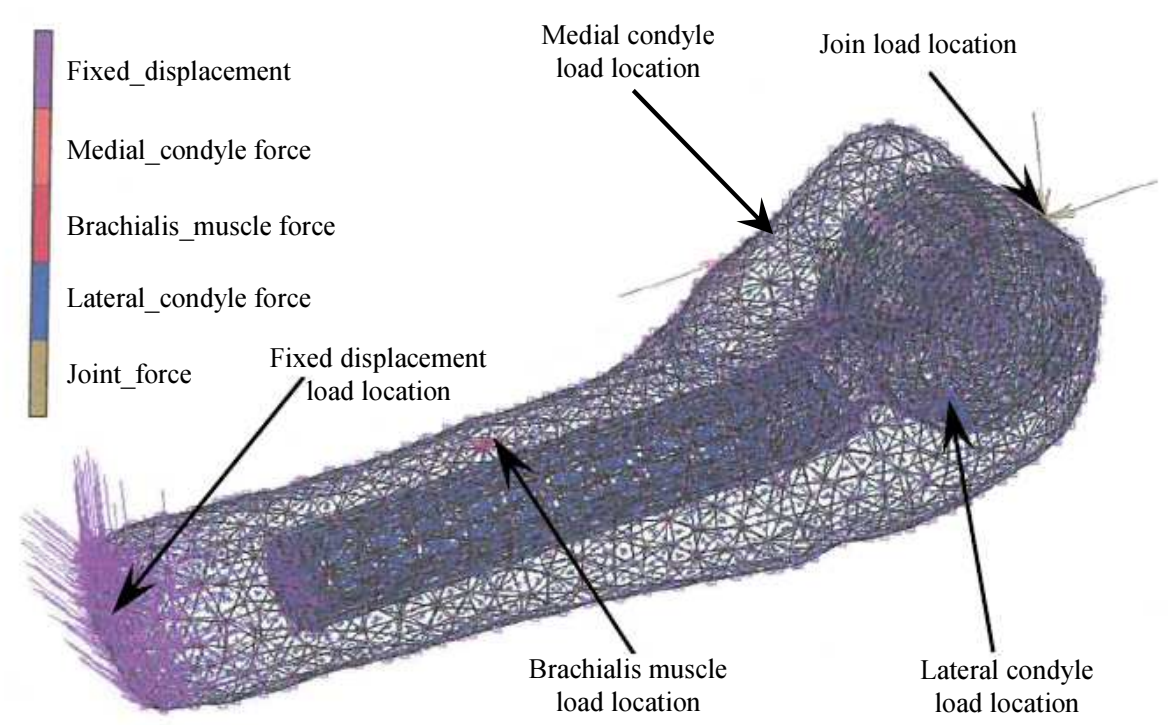

Fig. 24: Merged model indicating the points of application of the boundary conditions

Table 1: Data for the humeral cut-out model

\begin{tabular}{llll}
\hline & Length of implant model & \\
& --inch & 3-inch & 4-inch \\
\hline Number of elements & 8505 & 8501 & 8552 \\
Volume $\left(\mathrm{mm}^{3}\right)$ & 63515 & 60190 & 57656 \\
Surface $\operatorname{area}\left(\mathrm{mm}^{2}\right)$ & 15972 & 17036 & 17843 \\
\hline
\end{tabular}

Table 2: Data for the implant model

\begin{tabular}{llll}
\hline & Length of implant model & \\
& - - -inch & 3 -inch & 4-inch \\
\hline Number of elements & 3441 & 3239 & 3360 \\
\hline
\end{tabular}

Table 3: Data for the cement-mantle model

\begin{tabular}{llll}
\hline & \multicolumn{3}{l}{ Length of implant model } \\
& 2-inch & 3-inch & 4-inch \\
\hline Number of elements & 2019 & 2319 & 2740 \\
\hline
\end{tabular}

Table 4: Data for the cement-mantle model

\begin{tabular}{lllll}
\hline & $\begin{array}{l}\text { Lateral } \\
\text { condyle } \\
\text { load } @ 60^{\circ}\end{array}$ & $\begin{array}{l}\text { Medial } \\
\text { condyle load } \\
\text { load@ } 60^{\circ}\end{array}$ & $\begin{array}{l}\text { Brachialis } \\
\text { muscle } \\
\text { load @ } 43^{\circ}\end{array}$ & $\begin{array}{l}\text { Joint } \\
\text { load, } \\
@ 43^{\circ}\end{array}$ \\
\hline Point load $(\mathrm{kN})$ & 0.365 & 0.300 & 0.415 & 1.000 \\
\hline
\end{tabular}

External load in hand $\approx 0.067 \mathrm{kN}$

Table 5: Material properties

\begin{tabular}{llll}
\hline Model type & Material type & $\begin{array}{l}\text { Young's } \\
\text { modulus (GPa) }\end{array}$ & $\begin{array}{l}\text { Poison } \\
\text { ratio }\end{array}$ \\
\hline Cement-mantle & PMMA bone cement & 2 & 0.23 \\
Humerus & Cortical bone & 17 & 0.35 \\
Implant & Ti6Al4V alloy & 110 & 0.30 \\
\hline
\end{tabular}

\section{Contact}

The following deformable contact bodies with a coefficient of friction of 0.3 were set for the analysis, between:

- Humerus bone and the cement-mantle models

- Cement-mantle and the implant models

- Implant and the humerus bone models

\section{Analysis and Solution}

Considering the whole model as a non-linear static mechanical problem, the analysis was performed, by creating a load-case that contains the boundary conditions table and the contact bodies tables created. This load-case selected have constant time step of 0.02 and number of steps of 50. The Newton-Rhapson iteration scheme was used to apply the load to converge to the equilibrium state, within the specified tolerance of 0.001 . A convergence test was carried out to use for the iteration process, by plotting the peak value of maximum principal stress against the number of iterations, using the 3-inch implant model, Fig. 25. After solution has converged, the equivalent von Mises and the maximum principal stress distributions were obtained. Various results were analyzed, as discussed in the next section. Figure 26 shows a flowchart that gives a summary of the basic procedure applied in developing the elbow joint FE model and conducting the stress analysis. 


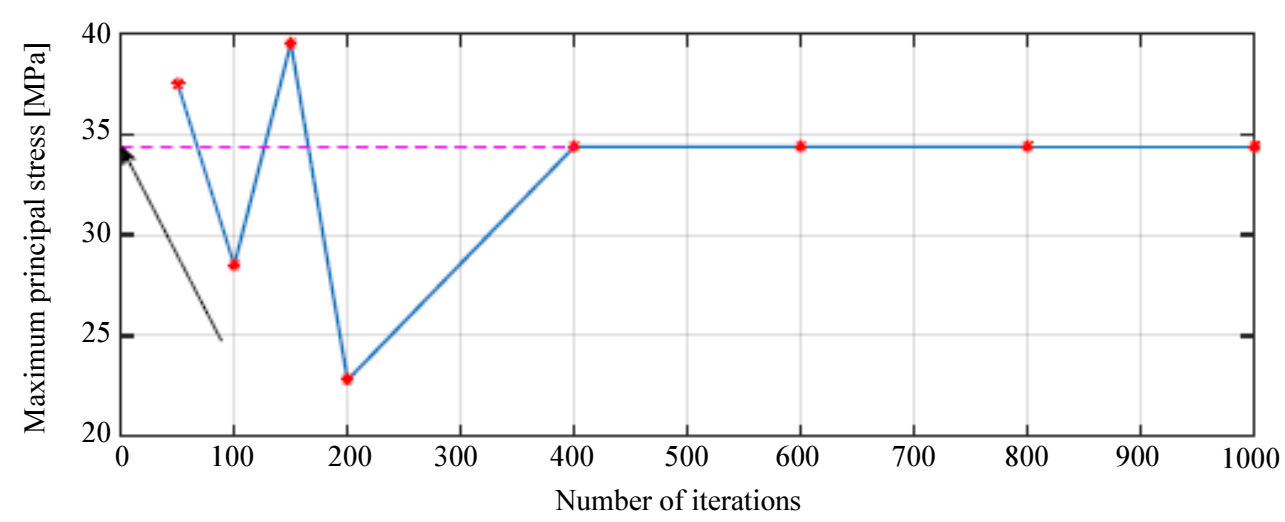

Fig. 25: Convergence test on the number of steps to use for the iteration process

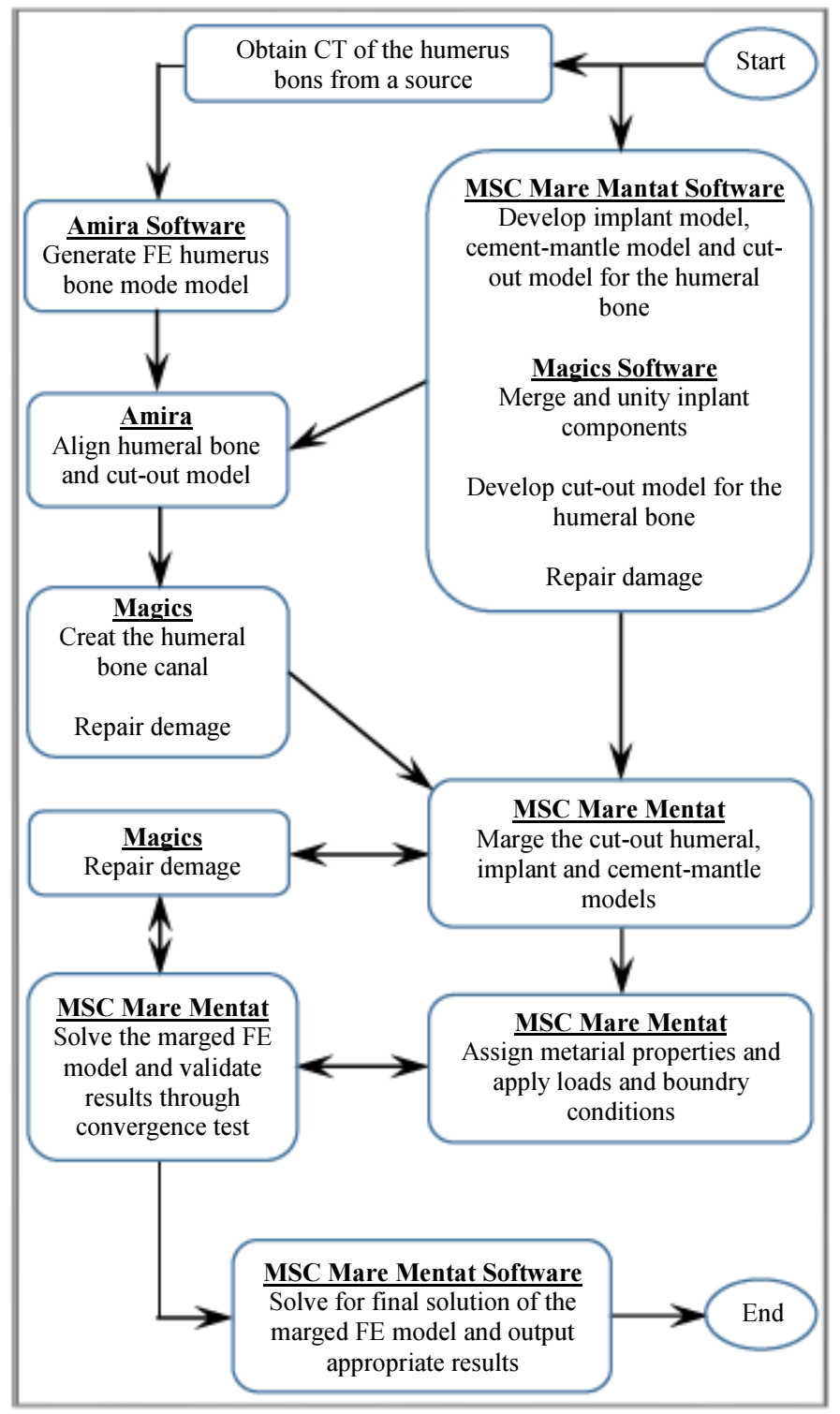

Fig. 26: Flowchart showing summary of the basic procedure used for the FEA of the elbow joint 


\section{Results and Discussion}

Figures 27-37 show maximum principal stresses and von Mises stress distributions obtained using the three implant stems. In general, the results indicate that the peak values of maximum principal stress or von Mises stress occurred at one location on the humerus bone. This is where failure of the bone will occur. The highest value of the three peak maximum principal stresses are shown Table 6 and Fig. 38.
This result indicates that as the length of the implant stem increases the peak stress on the humeral bone decreases. However, longer implant stems are not desirable and currently the 4-inch implant are the most widely used, even though 6 and 8 inch implant stems are also available. The result also indicates high stress concentration on the implant at the spots where the loads were applied; at the tip of the implant and around the neck of the implant. These stress distributions were expected.

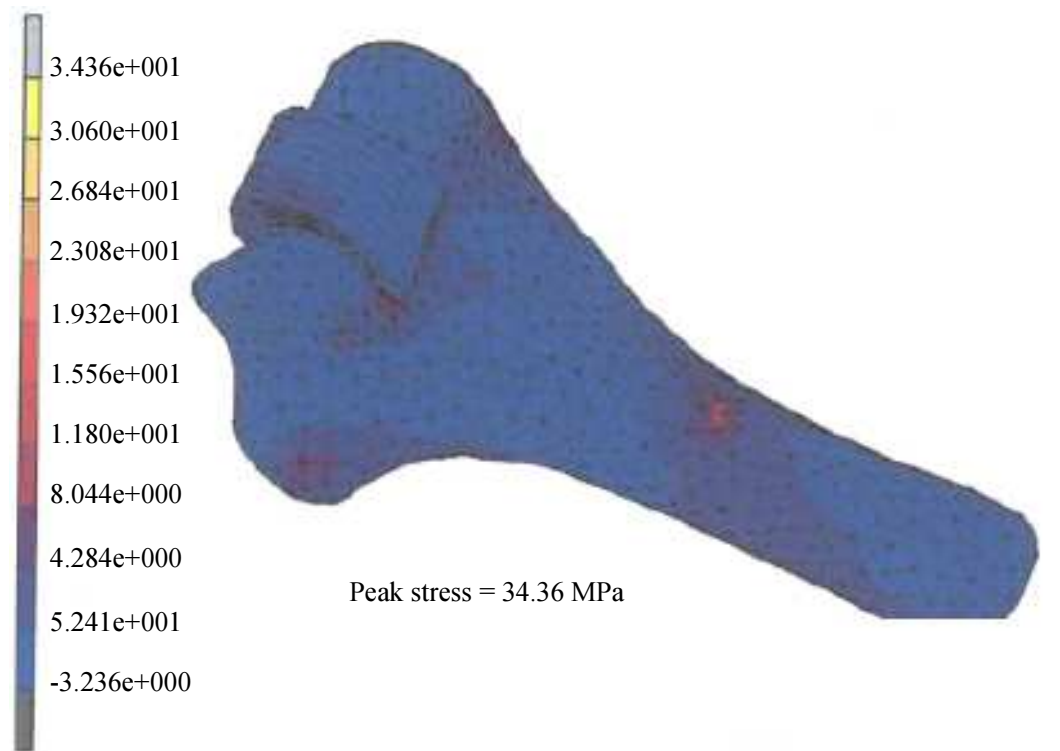

Fig. 27: Maximum principal stress distribution on the merged model: 3 -inch implant stem

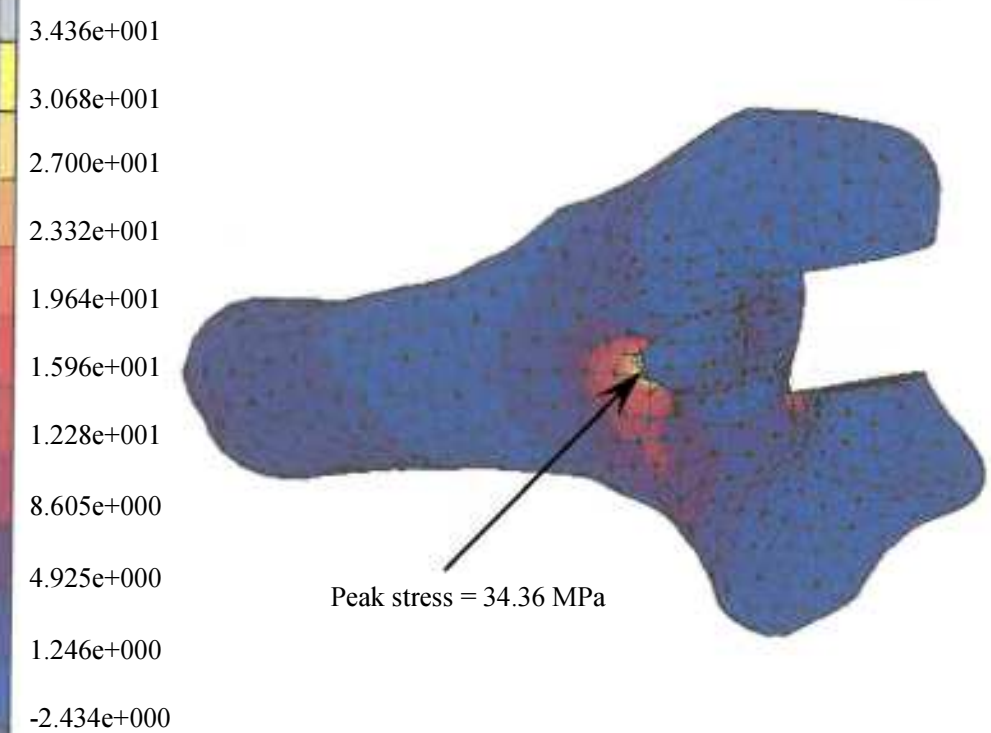

Fig. 28: Maximum principal stress distribution on the humerus model: 3-inch implant stem 


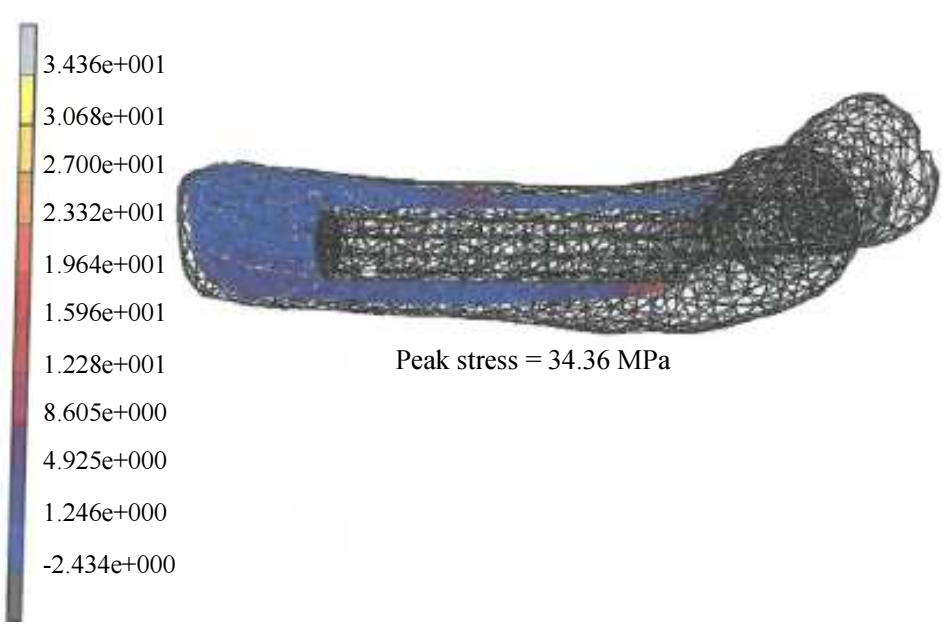

Fig. 29: Maximum principal stress distribution of the sagittal section on the humerus model: 3 -inch implant stem

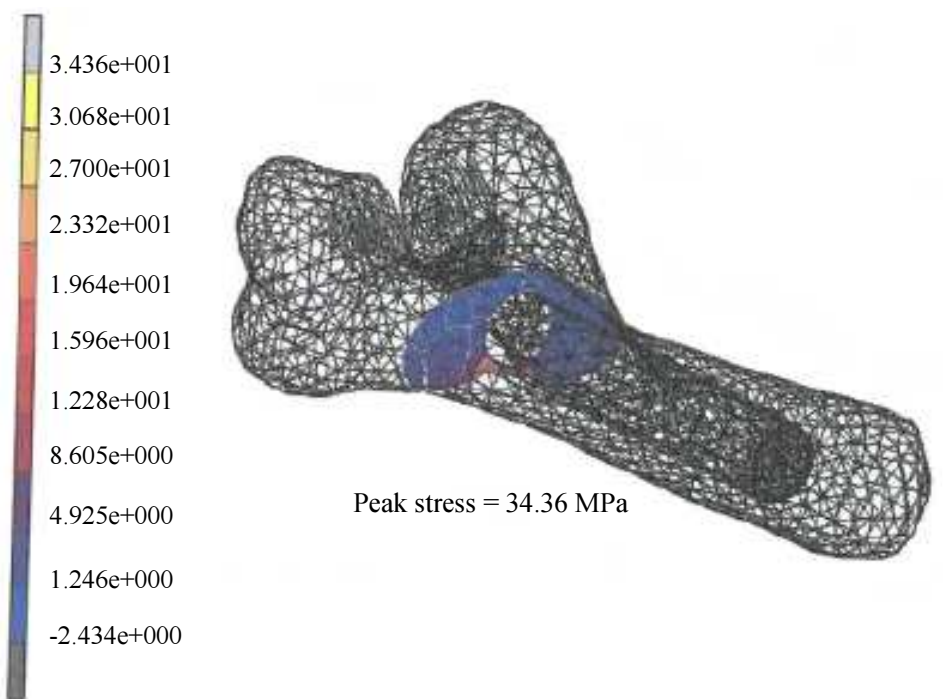

Fig. 30: Maximum principal stress distribution of the frontal section on the humerus model: 3-inch implant stem

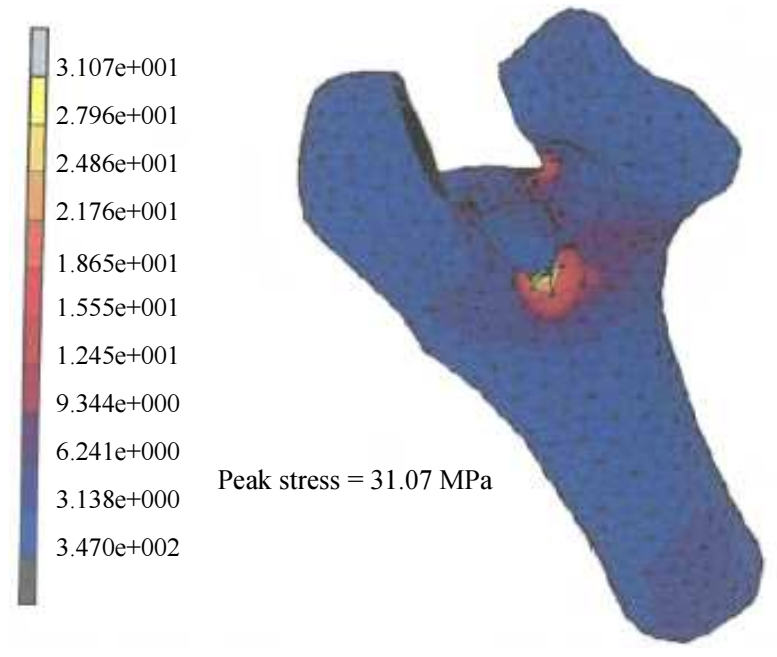

Fig. 31: von Mises stress distribution on the humerus model: 3-inch implant stem 


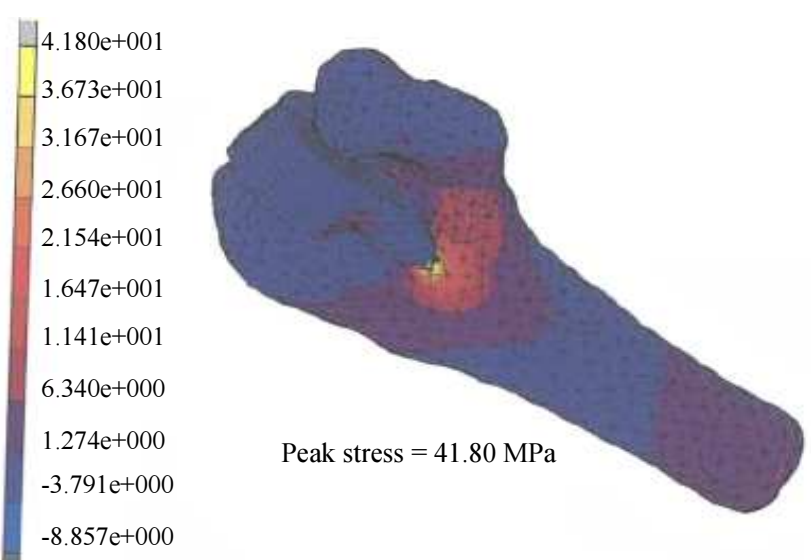

Fig. 32: Maximum principal stress on the humerus model: 2-inch implant stem
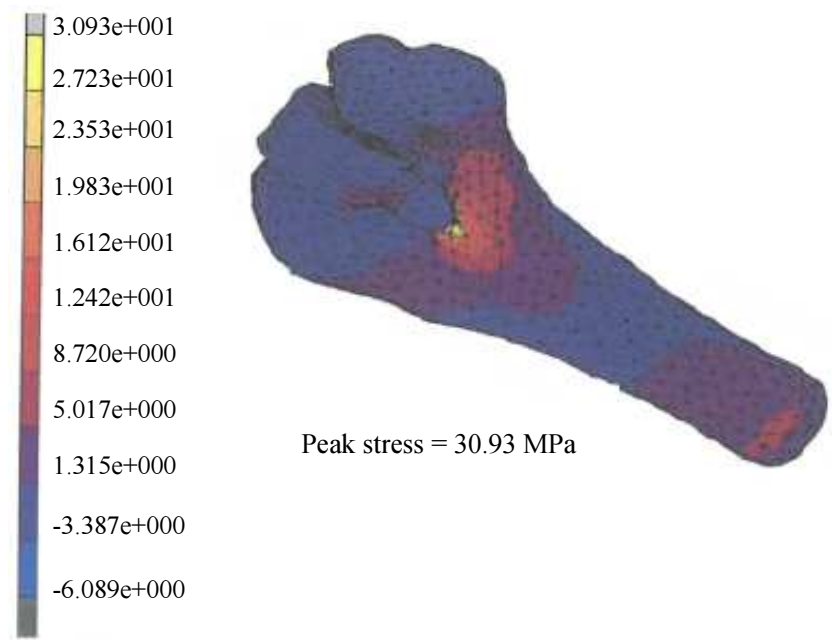

Fig. 33: Maximum principal stress on the merged model: 4-inch implant stem

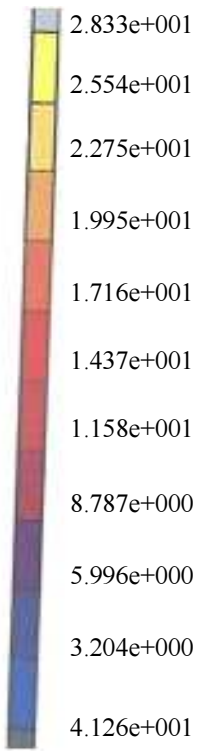

Fig. 34: von Mises stress on the 2-inch implant stem 


$$
\begin{aligned}
& 1.738 \mathrm{e}+001 \\
& 1.568 \mathrm{e}+001 \\
& 1.398 \mathrm{e}+001 \\
& 1.229 \mathrm{e}+001 \\
& 1.059 \mathrm{e}+001 \\
& 8.886 \mathrm{e}+000 \\
& 7.186 \mathrm{e}+000 \\
& 5.487 \mathrm{e}+000 \\
& 3.787 \mathrm{e}+000 \\
& 2.088 \mathrm{e}+000 \\
& 3.883 \mathrm{e}+001
\end{aligned}
$$

Fig. 35: von Mises stress on the 3-inch implant stem

\begin{tabular}{|l}
$-2.502 \mathrm{e}+001$ \\
$2.254 \mathrm{e}+001$ \\
$2.007 \mathrm{e}+001$ \\
$1.759 \mathrm{e}+001$ \\
$1.512 \mathrm{e}+001$ \\
$1.264 \mathrm{e}+001$ \\
$1.016 \mathrm{e}+001$ \\
$7.688 \mathrm{e}+000$ \\
$5.212 \mathrm{e}+000$ \\
$2.735 \mathrm{e}+000$ \\
$2.592 \mathrm{e}+001$
\end{tabular}

Fig. 36: von Mises stress on the 4-inch implant stem

$$
\begin{aligned}
& 8.921 \mathrm{e}+001 \\
& 7.572 \mathrm{e}+001 \\
& 6.224 \mathrm{e}+001 \\
& 4.875 \mathrm{e}+001 \\
& 3.527 \mathrm{e}+001 \\
& 2.178 \mathrm{e}+001 \\
& 8.300 \mathrm{e}+002 \\
& -5.185 \mathrm{e}+002 \\
& -1.867 \mathrm{e}+001 \\
& -3.215 \mathrm{e}+001 \\
& -4.564 \mathrm{e}+001
\end{aligned}
$$
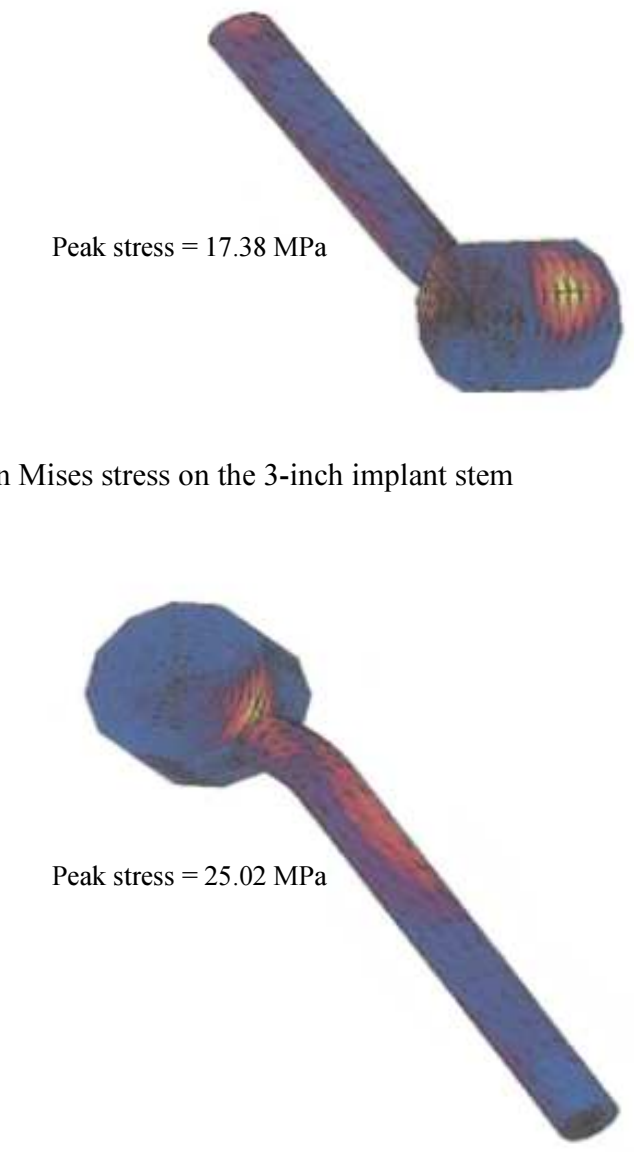


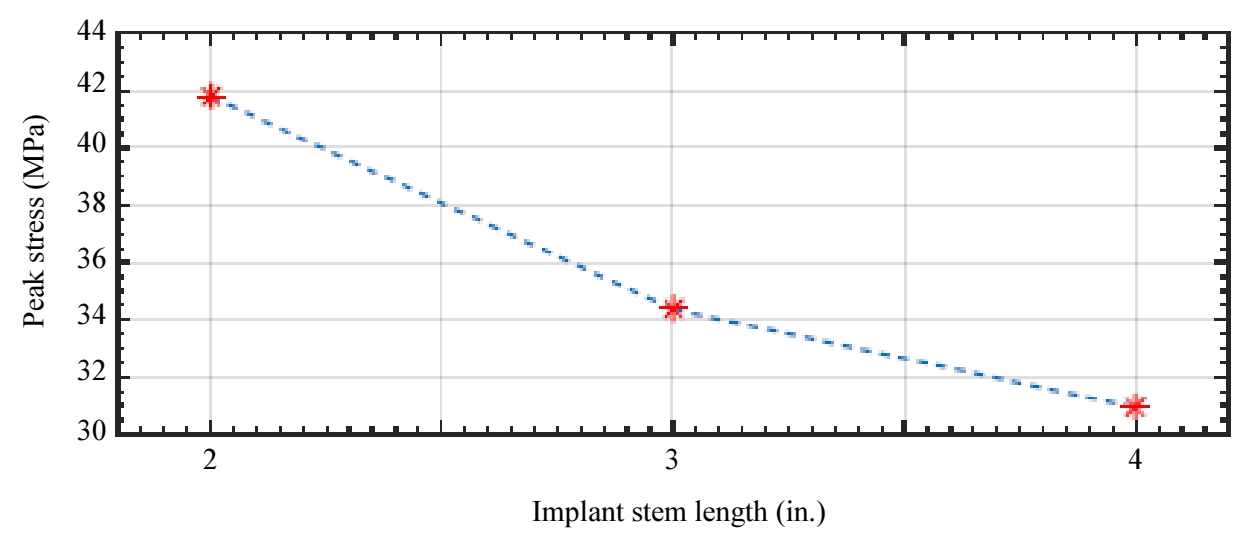

Fig. 38: Plot of the peak maximum principal stress against the length of implant stem

Table 6: Peak maximum principal stresses on the humerus bone for the various implant stem lengths

\begin{tabular}{ll}
\hline Implant stem length (in.) & $\begin{array}{l}\text { Peak maximum } \\
\text { principal stress }(\mathrm{MPa})\end{array}$ \\
\hline 2 & 41.80 \\
3 & 34.36 \\
4 & 30.93 \\
\hline
\end{tabular}

From Table 6, the peak stress values were low. Failure is not expected at this stress concentration spot under these peak tensile stresses for the various implants stems. Peak stress values between 100-200 MPa were expected with the $1.0 \mathrm{kN}$ joint load. A joint load between $1.2-3.0 \mathrm{kN}$ is expected to cause failure of the humerus bone initiated from the location of the peak maximum principal stress.

\section{Conclusion and Future Work}

\section{Conclusion}

The paper has demonstrated the application of software packages in the development of FEA humerus bone model from CT scan and implant-cement-mantle models from geometrical entities. Then, FEA conducted on the merged humerus, implant and cement models to obtain the stress distribution of the elbow joint. In general, the maximum principal stress and the equivalent von Mises stress obtained from the analysis, correlated well with the predictions and other research work. However, quantitatively, the stress values were low. Peak stress values between 100-200 MPa were expected with the 1.0 $\mathrm{kN}$ applied joint load. A joint load between 1.2-3.0 $\mathrm{kN}$ is expected to cause failure of the humerus bone initiated from the location of the peak maximum principal stress.

Overall, this work has shown that the FEA technique is a very powerful and cost-effective method to analyze the fixation of artificial human elbow joints into the bones of the human arm to a great degree of accuracy and flexibility. The same procedure can be extended to the other two bones at the elbow joint; radius and ulna. The interface stresses obtained can be of benefit to the design industry of elbow prosthesis and the orthopaedic surgeons in terms of material properties and length of stem of the implant to be used for specific applications.

\section{Future Work}

A more accurate stress distribution could be achieved. Further work on the subject is recommended and can be focussed in the following areas. First, a practical implant model needs to be developed instead of the simple cylindrical shape of the implant-stem and the implanthead used for this work. This can be done by importing a contour plot or the STL file of a practical implant into the FEA software and developing the mesh. Another way is to get very accurate dimensions of a practical implant taking into consideration all contours and features on it. One very important feature, which must be added in future work is the plasma spray coating, which enhances the cement interface for long-term fixation.

Second, a convergence test could be performed on the number of elements to be used for better mesh of the merged model, instead of using the maximum peak stress employed in this paper. Using the optimised number of elements will achieve a better mesh for satisfactory results.

Moreover, longer stem of the humerus bone is needed. The humerus bone model used for this project work was approximately $135 \mathrm{~mm}$ and having a longer one, a length of about $180 \mathrm{~mm}$ will give more accurate results. This will also give room for longer implant stems of 6 and 8 inch to be included in the analysis.

On final note, the biological material behaviour of the humerus bone should be used in the generation of the bone model. Bone operates on a micro-cellular level and as such many gross assumptions about the reaction of the material to loading and other stimuli have to be made. From the perspective of this work, the simulation of the behaviour was done on the macroscopic scale and was assumed to be isotropic and elastic material. Thus, was 
not subjected to the influence of the patient's genetic pre-disposition to any of the associated failures or diseases of bone such as osteolysis generated from wear debris. Developing realistic representation of bone material behaviour will allow the application of material properties directly to bone from CT scans.

\section{Acknowledgement}

I would like to express my gratitude to Professor A. A. Amis, Biomechanics Group, Department of Mechanical Engineering, Imperial College, London, UK.

\section{Ethics}

No part of this article may be reproduced without written permission from the publisher or author.

\section{References}

Alexander, R.M. and A. Vernon, 1975. The dimensions of knee and ankle muscles and the forces they exert. J. Human Movement Stud., 1: 115-123.

Amini, A.R., J.S. Wallace and S.P. Nukavarapu, 2011. Short-term and long-term effects of orthopedic biodegradable implants. J. Long-Term Effects Med. Implants.

Amis, A.A. and J.H. Miller, 1981. Elbow prosthesis. US Patent No. 4,242,758, Patent and Trademark Office, Washington, DC.

Amis, A.A., D. Dowson and V. Wright, 1979. Muscle strengths and musculoskeletal geometry of the upper limb. Eng. Med., 8: 41-48.

Amis, A.A., D. Dowson and V. Wright, 1980a. Elbow joint force predictions for some strenuous isometric actions. J. Biomechan., 13: 765-775.

Amis, A.A., D. Dowson and V. Wright, 1980b. Analysis of elbow forces due to high-speed forearm movements. J. Biomechan., 13: 825-831.

Barbour, K.E., 2017. Vital signs: Prevalence of doctordiagnosed arthritis and arthritis-attributable activity limitation-US, 2013-2015. Morbidity Mortality Weekly Report, 66: 246-253.

Basmajian, J.V. and A. Latif, 1957. Integrated actions and functions of the chief flexors of the elbow. J. Bone Joint Sur. Am., 39: 1106-1118.

Bone, 2017. https://en.wikipedia.org/wiki/Bone.

Brekelmans, W.A.M., H.W. Poort and T.J.J.H. Slooff, 1972. A new method to analyse the mechanical behaviour of skeletal parts. Acta Orthopaedica Scandinavica, 43: 301-317.

Completo, A., J. Pereira, F. Fonseca, A. Ramos and C. Relvas et al., 2011. Biomechanical analysis of total elbow replacement with unlinked iBP prosthesis: An in vitro and finite element analysis. Clinical Biomechanics, 26: 990-7.
Daripa, R., S. Majumder and A. Roychowdhury, 2004. Finite element analysis of total elbow prosthesis. Proceedings of the 5th Combined Meeting of the Orthopaedic Research Societies, (ORS’ 04).

EJ, 2017.2 The elbow joint. http://teachmeanatomy.info/upperlimb/joints/elbow-joint/.

EJP, 2017. Elbow joint prostheses. http://www.nakashima.co.jp/medical/en-iryo.html

EP, $2017 . \quad$ Elbow products. http://www.zimmer.com/medical-professionals.html

FEM, 2017. Finite element method. https://en.wikipedia.org/wiki/Finite_element method.

Hollerbach, J.M. and T. Flash, 1982. Dynamic interactions between limb segments during planar arm movement. Biol. Cybernet., 44: 67-77.

Hootman, J.M. and C.G. Helmick, 2006. Projections of US prevalence of arthritis and associated activity limitations. Arthritis Rheumatol., 54: 226-229.

Hopkins, A.R., U.N. Hansen, A.A. Amis, L. Knight and M. Taylor et al., 2007. Wear in the prosthetic shoulder: Association with design parameters. J. Biomech. Eng., 129: 223-230.

Khoo, T.A., M. Normahira, R. Revati, M.A. Majid and N.M. Nasir, 2006. A finite element analysis of elbow joint in daily activities. ARPN J. Eng. Applied Sci.

Kim, S., 2014. Contact stress analysis of the native radial head and radial head implants. $\mathrm{PhD}$ Thesis University of Pittsburgh.

Lawrence, S. and S. Gerald, 1963. Biomechanical power generated by forearm flexion 1. Human Factors, 5: 443-452.

Sanchez-Sotelo, J., 2016. Elbow rheumatoid elbow: Surgical treatment options. Current Rev. Musculoskeletal Medicine, 9: 224-231.

Schneeberger, A.G., D.C. Meyer and E.H. Yian, 2007. Coonrad-Morrey total elbow replacement for primary and revision surgery: A 2-7.5-year followup study. J. Shoulder Elbow Surgery, 16: S47-S54.

Shaw, G., 2017. Hip and knee replacements on the rise. http://www.webmd.com/arthritis/features/hip-kneereplacements-rise $\# 1$.

Studer, A. and G.S. Athwal, 2011. Rheumatoid arthritis of the elbow. Hand Clin., 27: 139-150.

Tidy, C., 2017. Elbow joint replacement. https://patient.info/doctor/elbow-joint-replacement

Wadsworth, T.G., 1982. The Elbow. 2nd Edn., Churchill Livingstone, Edinburgh, New York, ISBN-10: 0443019312, pp: 354.

Witte, F., 2015. Reprint of: The history of biodegradable magnesium implants: A review. Acta Biomaterialia, 23: S28-S40.

Zuckerman, J.D. and F.A. Matsen, 1989. Biomechanics of the elbow. Basic Biomechanics of the Musculoskeletal System, pp: 35-46. 